\title{
Clinical Efficacy of Traditional Chinese Medicine, Suan Zao Ren Tang, for Sleep Disturbance during Methadone Maintenance: A Randomized, Double-Blind, Placebo-Controlled Trial
}

\author{
Yuan-Yu Chan, ${ }^{1,2}$ Yi-Hung Chen, ${ }^{3}$ Szu-Nian Yang, ${ }^{1}$ Wan-Yu Lo, ${ }^{4}$ and Jaung-Geng Lin ${ }^{5}$ \\ ${ }^{1}$ Department of Psychiatry, Taoyuan Armed Forces General Hospital, No. 168, Zhong-Xing Road, Taoyuan 32551, Taiwan \\ ${ }^{2}$ Graduate Institute of Integrated Medicine, China Medical University, No. 91, Hsueh-Shih Road, Taichung 40402, Taiwan \\ ${ }^{3}$ Graduate Institute of Acupuncture Science, China Medical University, No. 91, Hsueh-Shih Road, Taichung 40402, Taiwan \\ ${ }^{4}$ Department of Biotechnology, Hung Kuang University, No. 1018, Sec. 6, Taiwan Boulevard, Shalu District, Taichung 43302, Taiwan \\ ${ }^{5}$ Graduate Institute of Chinese Medical Science, China Medical University, No. 91, Hsueh-Shih Road, Taichung 40402, Taiwan
}

Correspondence should be addressed to Wan-Yu Lo; drwanyu@gmail.com and Jaung-Geng Lin; jglin@mail.cmu.edu.tw

Received 4 December 2014; Revised 7 July 2015; Accepted 22 July 2015

Academic Editor: Veronique Seidel

Copyright (C) 2015 Yuan-Yu Chan et al. This is an open access article distributed under the Creative Commons Attribution License, which permits unrestricted use, distribution, and reproduction in any medium, provided the original work is properly cited.

Methadone maintenance therapy is an effective treatment for opiate dependence, but more than three-quarters of persons receiving the treatment report sleep quality disturbances. In this double-blind, randomized, controlled trial, we recruited 90 individuals receiving methadone for at least one month who reported sleep disturbances and had Pittsburgh Sleep Quality Index (PSQI) scores $>5$. The purpose of this study was to determine whether Suan Zao Ren Tang, one of the most commonly prescribed traditional Chinese medications for treatment of insomnia, improves subjective sleep among methadone-maintained persons with disturbed sleep quality. Ninety patients were randomly assigned to intervention group $(n=45)$ and placebo group $(n=45)$, and all participants were analyzed. Compared with placebo treatment, Suan Zao Ren Tang treatment for four weeks produced a statistically significant improvement in the mean total PSQI scores $(P=0.007)$ and average sleep efficiency $(P=0.017)$. All adverse events (e.g., lethargy, diarrhea, and dizziness) were mild in severity. Suan Zao Ren Tang is effective for improving sleep quality and sleep efficiency among methadone-maintained patients with sleep complaints.

\section{Introduction}

Heroin dependence is one of the chronic medical illnesses and a public health problem worldwide. The social, medical, and economic consequences of heroin dependence are profound, including disrupted relationships, lost productivity, crime, violence, and the transmission of HIV/AIDS and other infectious diseases $[1,2]$. Since the mid-1960s, longterm methadone maintenance therapy (MMT) has been the treatment of choice for heroin dependence [3-6] and has proven to be an effective long-term treatment for severe opiate dependence [7]. However, more than three-quarters of persons receiving MMT report sleep quality disturbances [810]. The severity of sleep symptoms has been associated with comorbid psychiatric disorders, chronic pain, lower levels of daily functioning, and the use of other drugs [9-11].

Methadone-maintained patients with sleep disturbances receive, on average, fewer than six hours of sleep daily [12]. A study from China found that $96.3 \%$ of methadonemaintained patients had Pittsburgh Sleep Quality Index (PSQI) scores $\geq 8$ [13]. Other studies have reported that $84 \%$ of methadone-maintained patients have PSQI scores $\geq 6$ [10], whereas another study found that only $35.2 \%$ of the general population had PSQI scores $>5$ [14]. Generally, the incidence of sleep disturbances among methadone-maintained patients is significantly higher than that for the general population. Short sleep durations have been associated with daytime symptoms such as cognitive difficulties, reduced adherence to 
methadone treatment, and increased risk of relapse, physical injury, and motor-vehicle accidents $[15,16]$. Two polysomnographic studies show reduction of rapid eye movement (REM) sleep and slow wave sleep during induction and maintenance of opioid use $[17,18]$.

Sleep-disordered breathing, including obstructive sleep apnea (OSA) and central sleep apnea (CSA), is another potential factor leading to disrupted sleep in MMT patients. Some studies showed that CSA has been reported to occur in $30-60 \%$ of MMT patients $[19,20]$ and associated with methadone dose and concomitant benzodiazepine use [21].

In one study of 71 OSA and CSA patients who were in MMT at least 3 months, the results showed that CSA was not associated with methadone doses and OSA was more common than CSA in this population. OSA was associated with higher body mass index, longer duration in MMT, and non-Caucasian race. Moreover, subjective sleep disturbance measured with the PSQI was not related to OSA or CSA [12].

There are some mechanisms that may explain insomnia among methadone-maintained patients. First, opioids decrease REM sleep by decreasing acetylcholine release in the pontine reticular formation [22]. Second, opioids promote wakefulness by suppressing inhibitory gamma-aminobutyric acid (GABA) transmission in the dorsal raphe nucleus [23]. Third, sleep disturbances in MMT patients are associated with opioid-induced reduction of the nucleoside adenosine in the basal forebrain $[24,25]$.

In Western medicine, therapy for insomnia is mainly based on prescribed medications such as benzodiazepines (BZD), anticonvulsants, antidepressants, or over-the-counter antihistamines [26]. These medications, especially BZD, are sometimes associated with adverse effects and are not suitable for long-term use due to an increased risk of life-threatening multiple drug overdoses. In opiate-dependent patients, the misuse and abuse of BZD is a public health problem because methadone and BZD both have sedating effects on the central nervous system, which lead to difficulty in breathing as well as cognitive impairment $[27,28]$. A recent survey revealed a $47 \%$ prevalence of lifetime use of BZD among methadonemaintained patients [29] and nonprescribed BZD use among MMT patients ranged from 44 to $66 \%[30,31]$. In addition, methadone-maintained patients who abuse BZD are associated with higher rates of depression and anxiety [32], increased risk of continuing opiate abuse, and discontinuing methadone treatment $[33,34]$.

Traditional Chinese medicine (TCM) has been widely used for centuries in Asian countries and is one of the most common complementary and alternative therapies used in Taiwan [35-38]. Previous studies have shown that TCM treatment is effective in improving sleep quality and prolonging sleep duration, and it is associated with fewer side effects (e.g., lethargy, dry mouth, and dizziness) than Western medicines [39-41].

In Taiwan, the National Health Insurance program has found that Suan Zao Ren Tang (SZRT) was the most commonly prescribed Chinese herbal formula for the treatment of insomnia [42]. SZRT was also the most common ingredient found in over-the-counter sleeping pills [43]. SZRT has been used to treat insomnia for centuries, as noted in the ancient Chinese book Synopsis of Prescriptions of the Golden Chamber, by Chang Chung Ching (AD 150-219). In classical literature, SZRT is said to bring on a tranquillizing sensation and assists in alleviating insomnia, restlessness, palpitations, and mental distress. In 2007, Yi et al. found SZRT was shown to increase non-REM sleep in an experimental model in rats, and the mechanism was thought to involve the stimulation of $\mathrm{GABA}_{\mathrm{A}}$ and serotonin receptors [44, 45].

Systematic reviews of complementary and herbal medicine for insomnia have been published recently [46-48], but these reviews found that a majority of the randomized clinical trials on TCM were of poor methodological quality. However, SZRT has never been tested in opiate-dependent patients. Therefore, we have designed a randomized, doubleblind, placebo-controlled clinical trial to test whether SZRT is effective in improving sleep quality, sleep latency, sleep duration, sleep efficiency, anxiety, and depression among methadone-maintained patients with sleep complaints.

\section{Methods}

2.1. Participants. Patients were recruited from the outpatients of the Department of Psychiatry at Taoyuan Armed Forces General Hospital, a regional teaching hospital in Taiwan, between August 2013 and December 2013. Ninety patients were recruited for the study and divided into an intervention group and a placebo group.

Patients eligible for inclusion in the study were over 20 years of age; had fulfilled the criteria for opiate dependence (Diagnostic and Statistical Manual of Mental Disorders, Fourth Edition); had been receiving MMT for more than one month; had reported a sleep disturbance with a PSQI score $>5$; and provided signed informed consent. Individuals were excluded if they had received any antidepressant or neuroleptic medication; had received any TCM treatment during the previous 30 days; had any serious physical or mental illness; exhibited a significant risk of suicide; were pregnant; or were unable to read and fill out the forms for the study.

2.2. Study Design and Procedure. The study was conducted as a randomized, double-blind, placebo controlled trial to examine the effects of SZRT in methadone-maintained persons with sleep complaints. All participants were randomly assigned to an intervention or placebo group in a ratio of 1:1 using computer generated random numbers without stratification by background characteristics. The random number list was prepared by an investigator with no clinical involvement in the trial. The allocation sequence was concealed from the researcher enrolling and assessing participants in sequentially numbered, opaque, sealed, and stapled envelopes. Corresponding envelopes were opened only after the enrolled participants completed all assessments and intervention. The research staff and participants were blinded to their assignment. The study procedures and informed consent form were approved by the Institutional Review Board of Tri-Service General Hospital National Defense Medical Center in Taiwan (IRB number TY101-18) and registered in http://www.clinicaltrials.gov/ (NCT01913418). 
2.3. Herbal Formula. In a typical Chinese herbal medicine prescription, a complex integration of two or more single Chinese herbs is formulated together to achieve additive or synergistic effects. SZRT is composed of the following five herb ingredients: Semen Zizyphi Spinosae (Suanzaoren), Sclerotium Poriae Cocos (Fuling), Radix Ligustici Chuanxiong (Chuanxiong), Rhizoma Anemarrhena (Zhimu), and Radix Glycyrrhizae (Gancao). The SZRT formula used in this study was manufactured as a herbal extract powder using the good manufacturing procedures of the certified company Kaiser Pharmaceutical Co., Ltd. (Taiwan). The granules were packed in aluminum foil packages and administered orally at a dose of $4 \mathrm{~g}$, three times per day for four weeks. Kaiser Pharmaceutical was not involved in any other sponsorship, study design, or monitoring of the participants. The placebo granules were prepared with $4 \mathrm{~g}$ starch inside foil packages of the same color and size as the SRZT. Thus, neither the investigators nor patients knew the group to which a patient was allocated.

2.4. Intervention. The participants were provided either SZRT or placebo and the intervention period was four weeks. We selected the intervention period because of a previous study that reported a significant improvement of sleep quality after four weeks of SZRT treatment [49]. In each week, patients were provided with a one-week supply of SZRT or placebo. No other medication or behavioral therapy to address the sleep disturbance was allowed during the study period. After four weeks on the prescribed medication, the participants discontinued the use of SZRT or placebo.

2.5. Measures. Patient questionnaires included basic data such as gender, age, marital status, occupation, recent heroin use, recent amphetamine use, history of heroin use, and history of amphetamine use. The patients completed the questionnaire at baseline (first visit) and after four weeks (second visit). In this study, all assessments were completed by the same rater who was blind to the treatment allocation.

The primary outcome measure under investigation was sleep quality. Sleep quality was assessed using the Taiwanese version of the PSQI [50], which has demonstrated reliability and validity [51]. This system evaluates sleep disturbances in seven subscales: subjective sleep quality, sleep latency, sleep duration, habitual sleep efficiency, sleep disturbances, use of sleeping medication, and daytime dysfunction. Each subscale is rated on a 4-point scoring system ( 0 to 3 , with 3 indicating a more profound effect), and scores from the subscales are summed together to yield a global score (0 to 21). Higher scores indicate a greater severity of sleep disturbance, and a global score $>5$ is indicative of "poor sleep" [50].

Secondary outcome measures include one-week sleep diaries, severity of Beck anxiety inventory (BAI) score, Beck Depression Inventory II (BDI-II, second edition) score, and heroin craving. Participants completed a daily morning sleep diary during the week in which they recorded bedtime, time to fall asleep, number of awakenings, time awake during the night, and wake-up time. Total time in bed was calculated as the duration between bedtime and wake-up time. Diary total sleep time (TST) was calculated by subtracting sleep onset latency (SOL) and time awake during the night from diary time in bed. Diary sleep efficiency (SE) was calculated by dividing diary total sleep time by total time in bed $\times 100$. Each sleep measure was averaged over the reported days. We included participants with 3 to 7 diary days because sleep diary analyses in other populations indicate that reliable sleep estimates can be obtained with $\geq 3$ days of data [52].

The severity of anxiety symptoms was measured using the BAI [53]. This measure lists 21 symptoms of anxiety, such as feeling hot, scared, or nervous. Participants were instructed to rate the extent to which they were bothered by each of these symptoms over the previous week. Each item could be rated on a 4-point Likert scale, ranging from 0 (not bothered) to 3 (severely bothered), yielding a maximum total score of 63 points. The BAI manual considers total scores of 0 through 7 minimal anxiety, 8 through 15 mild anxiety, 16 through 25 moderate anxiety, and 26 through 63 severe anxiety.

Depression symptoms were measured using the BDI-II. This measure consists of 13 items, rated from 0 to 3 , to evaluate depression according to the degree to which it reflected the patient's state during the previous week. The BDI-II has a high reliability and concurrent validity and the BDI-II manual considers total scores of 0 through 13 minimal depression, 14 through 19 mild depression, 20 through 28 moderate depression, and 29 through 63 severe depression [54].

The severity of heroin craving was assessed using a 100millimeter visual analog scale (VAS). The amount of heroin craving experienced by a participant can range across a continuum from no craving to extreme craving. The VAS score was determined by measuring in millimeters from the left-hand end of the line to the point that the patient marks [55].

2.6. Statistical Analysis. Statistical analyses were based on intention-to-treat model. Missing data was handled using last observation carried forward imputation technique. SPSS (version 18.0) was used for statistical analysis. Baseline differences and the treatment effects between the SZRT and the placebo group were examined using either unpaired $t$ tests or $\chi^{2}$ tests.

Our sample size estimation was based on changes in PSQI score, the primary outcome measure. Based on a previous SZRT study [49], a clinically significant treatment effect was defined as $\geq 2$ point difference in PSQI score between treatment groups, and the effect size was 0.667 . G* Power 3.1.3 was used for $t$-tests to compute that a sample of 37 individuals per group would have a power of $80 \%$ to differentiate between groups at an alpha level 0.05 . Allowing for a $20 \%$ attrition rate, we estimated this study would require a sample size of 45 patients per group.

\section{Results}

3.1. Patient Characteristics. Of 175 potential patients assessed for eligibility, 85 declined participation or failed to meet inclusion criteria (Figure 1). Ninety patients were randomly assigned to the intervention group $(n=45)$ and the placebo group $(n=45)$, while $80(88.9 \%)$ of them completed the 4 week treatment. The mean age of participants was 39.6 years, 


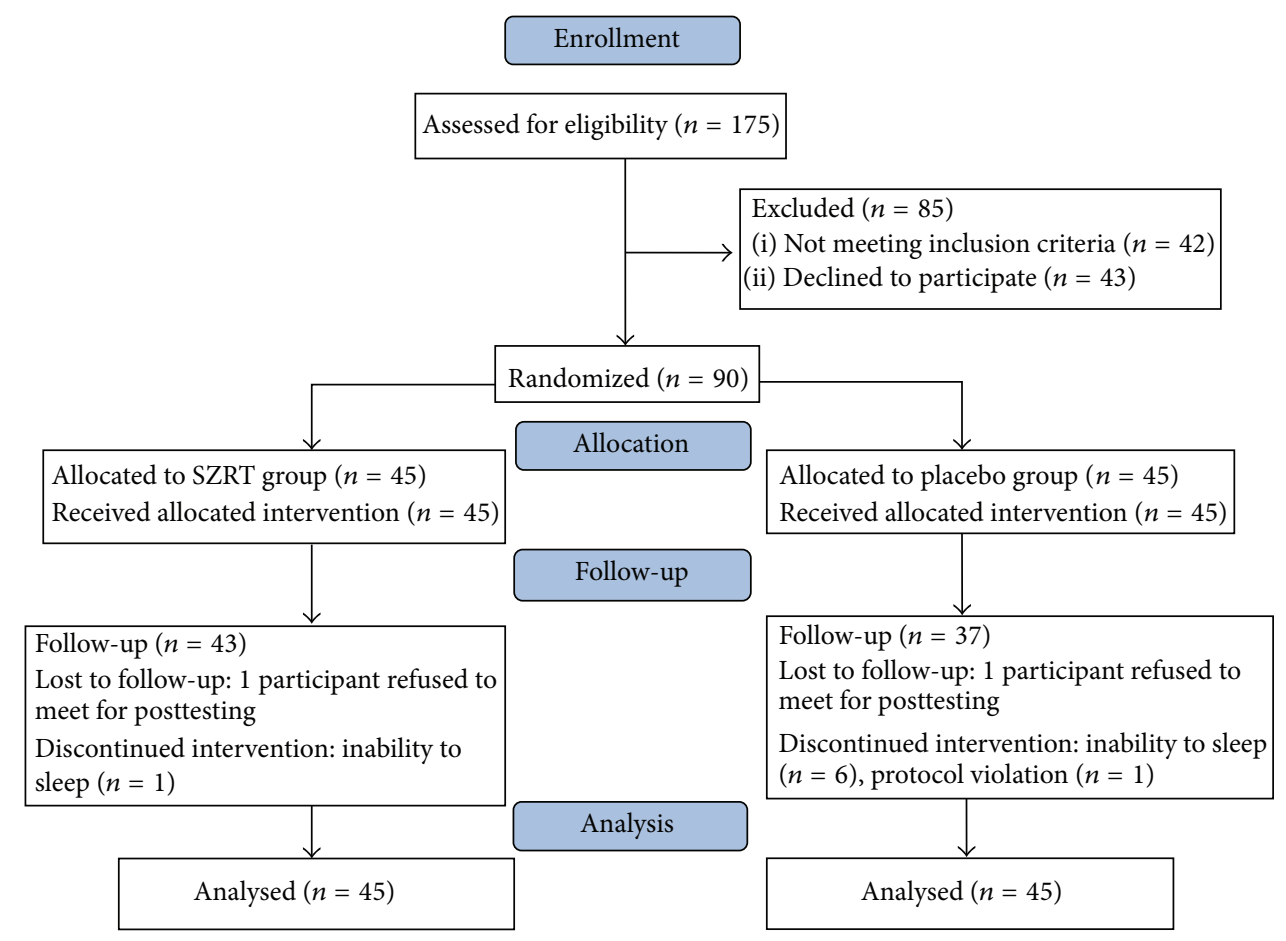

FIGURE 1: The diagram specifics the flow of participants through the enrollment, allocation, follow-up, and analysis phases of the RCT study.

and $80 \%$ were male (Table 1 ). The mean PSQI total score was $11.5 \pm$ 3.1. Mean methadone dose at baseline was $55.7 \pm$ $31.6 \mathrm{mg}$. Heroin and amphetamine abuse histories averaged $9.4 \pm 5.7$ and $5.5 \pm 6.5$ years, respectively. In the 30 days prior to baseline, $53.3 \%$ and $20 \%$ of participants reported using heroin or amphetamine, respectively. Intervention groups did not differ significantly $(P>0.05)$ in any of the baseline characteristics described in Table 1 . Seven subjects (7.8\%) discontinued intervention within the three weeks due to a complaint of no response after treatment. One subject (1.1\%) violated the protocol due to severe alcohol drinking and using other medication. Two subjects (2.2\%) lost to followup because they refused to meet for posttesting (Figure 1).

3.2. Efficacy. Table 2 shows baseline means, follow-up means, and changes in mean subjective sleep parameters by intervention group. The result shows that between baseline and week 4, mean total PSQI scores decreased by 3.8 and 1.9 points, and average SE increased by $11.4 \%$ and $5.0 \%$ among those randomized to SZRT and placebo, respectively. Compared with placebo treatment, Suan Zao Ren Tang treatment for four weeks produced a statistically significant improvement in the mean total PSQI scores $(P=0.007)$ and average SE $(P=0.017)$. In the SZRT group, the average time to fall asleep per day decreased from 41.5 minutes at baseline to 26.5 minutes after treatment. The average total sleep time per day increased from 342.6 minutes at baseline to 412.8 minutes after treatment.

Table 3 presents the mean values for secondary outcomes by treatment condition at baseline and after treatment. After treatment, BAI and BDI scores in both groups improved from the baseline. However, BAI, BDI, and heroin craving scores did not differ significantly between the groups after treatment.

3.3. Adverse Events. In the SZRT group, diarrhea $(n=2)$, sweating $(n=2)$, dizziness $(n=1)$, and morning sleepiness $(n=1)$ were reported as adverse events. In the placebo group, adverse events included morning sleepiness $(n=2)$ and headache $(n=1)$. Most adverse events were mild in severity.

\section{Discussion}

To the best of our knowledge, this is the first randomized, placebo-controlled trial testing SZRT for opiate-dependent persons, a population with extraordinarily high rates of sleep disturbances. Some studies suggest that poor-quality sleep is a universal risk factor for drug relapse [56], and it is possible that there are overlapping neurobiological mechanisms common to sleep disorders, depression, and anxiety $[57,58]$. Our results demonstrate that SZRT improves subjective sleep quality and sleep efficiency in methadonemaintained persons with sleep disturbances. But the PSQI score was still more than five at the end of treatment; longterm treatment effect should be investigated in the future.

In our study, we found that the chance of dropping out was higher for patients treated with placebo than for those treated with SZRT. There are six patients discontinued intervention because of inability to sleep in the placebo group. However, only one patient discontinued intervention because of the same reason in the SZRT group. A previous meta-analysis reported that attrition rate may be higher in 
TABLE 1: Demographic and clinical characteristics of the participants.

\begin{tabular}{|c|c|c|c|c|c|}
\hline Variable & SZRT $(n=45)$ & Placebo $(n=45)$ & Total $(n=90)$ & $\chi^{2} / t$ value $^{\mathrm{a}}$ & $P$ value \\
\hline Age (years) & $40.6 \pm 7.2$ & $38.6 \pm 6.9$ & $39.6 \pm 7.1$ & 1.34 & 0.18 \\
\hline Gender (male) & $34(75.6 \%)$ & $38(84.4 \%)$ & $72(80 \%)$ & 1.11 & 0.29 \\
\hline Duration of methadone maintenance (months) & $25.4 \pm 19.8$ & $19.6 \pm 19.7$ & $22.5 \pm 19.8$ & 1.40 & 0.17 \\
\hline Recent heroin use (yes) & $24(53.3 \%)$ & $24(53.3 \%)$ & $48(53.3 \%)$ & 0.00 & 1.00 \\
\hline Recent amphetamine use (yes) & $11(24.4 \%)$ & $7(15.6 \%)$ & $18(20 \%)$ & 1.11 & 0.29 \\
\hline Heroin use (years) & $8.8 \pm 5.7$ & $9.9 \pm 5.8$ & $9.4 \pm 5.7$ & -0.84 & 0.40 \\
\hline Amphetamine use (years) & $4.9 \pm 6.0$ & $6.1 \pm 6.9$ & $5.5 \pm 6.5$ & -0.85 & 0.40 \\
\hline Methadone dosage (mg) & $58.9 \pm 32.5$ & $52.4 \pm 30.6$ & $55.7 \pm 31.6$ & 0.97 & 0.34 \\
\hline PSQI total scores & $11.6 \pm 3.6$ & $11.5 \pm 2.5$ & $11.5 \pm 3.1$ & 0.10 & 0.92 \\
\hline Total time in bed (min) & $446.6 \pm 104.2$ & $489.8 \pm 111.5$ & $468.2 \pm 109.5$ & -1.90 & 0.06 \\
\hline Total sleep time (min) & $342.6 \pm 95.2$ & $377.0 \pm 101.1$ & $359.8 \pm 99.2$ & -1.66 & 0.10 \\
\hline Sleep efficiency $(\%)$ & $76.5 \pm 11.3$ & $79.0 \pm 11.1$ & $77.8 \pm 11.2$ & -1.05 & 0.30 \\
\hline Sleep onset latency (min) & $41.5 \pm 36.7$ & $38.8 \pm 18.9$ & $40.1 \pm 29.0$ & 0.45 & 0.66 \\
\hline BAI & $15.0 \pm 11.3$ & $12.4 \pm 13.9$ & $13.7 \pm 12.6$ & 0.96 & 0.34 \\
\hline BDI-II & $18.3 \pm 14.3$ & $14.4 \pm 14.2$ & $16.4 \pm 14.4$ & 1.27 & 0.21 \\
\hline Heroin craving score (mm) & $25.3 \pm 28.1$ & $33.7 \pm 34.6$ & $29.6 \pm 31.6$ & -1.22 & 0.22 \\
\hline
\end{tabular}

Note: data are presented as mean \pm SD or number (\%). ${ }^{\mathrm{a}}$ Comparison between SZRT group and placebo group by $\chi^{2}$ or unpaired $t$-test. PSQI, Pittsburgh Sleep Quality Index; BAI, Beck Anxiety Inventory; BDI-II, Beck Depression Inventory II.

TABLE 2: Mean and change in mean subjective sleep parameters.

\begin{tabular}{|c|c|c|c|c|c|}
\hline & \multicolumn{2}{|c|}{ SZRT group $(n=45)$} & \multicolumn{2}{|c|}{ Placebo group $(n=45)$} & \multirow{2}{*}{$P$ value ${ }^{\mathrm{a}}$} \\
\hline & Mean (SD) & Change & Mean (SD) & Change & \\
\hline \multicolumn{6}{|l|}{ PSQI } \\
\hline \multicolumn{6}{|l|}{ Total scores } \\
\hline Baseline & $11.6(3.6)$ & & $11.5(2.5)$ & & \\
\hline 4-week posttreatment & $7.8(3.7)$ & -3.8 & $9.6(3.1)$ & -1.9 & $0.007^{*}$ \\
\hline \multicolumn{6}{|l|}{ Sleep diary } \\
\hline \multicolumn{6}{|l|}{ Total time in bed } \\
\hline Baseline & $446.6(104.2)$ & & $489.8(111.5)$ & & \\
\hline 4-week posttreatment & $466.3(78.8)$ & 24.1 & $481.7(113.6)$ & -5.5 & 0.261 \\
\hline \multicolumn{6}{|l|}{ Mean TST } \\
\hline Baseline & $342.6(95.2)$ & & $377.0(101.1)$ & & \\
\hline 4-week posttreatment & $412.8(81.3)$ & 70.2 & $418.8(121.9)$ & 41.8 & 0.228 \\
\hline \multicolumn{6}{|l|}{ Mean SL } \\
\hline Baseline & $41.5(36.7)$ & & $38.8(18.9)$ & & \\
\hline 4-week posttreatment & $26.5(18.3)$ & -15.0 & $31.8(31.5)$ & -6.9 & 0.211 \\
\hline \multicolumn{6}{|l|}{ Mean SE (\%) } \\
\hline Baseline & $76.5(11.3)$ & & $79.0(11.1)$ & & \\
\hline 4-week posttreatment & $87.9(9.0)$ & 11.4 & $84.1(15.4)$ & 5.0 & $0.017^{*}$ \\
\hline
\end{tabular}

${ }^{a}$ Difference between mean change scores SZRT and placebo groups ( $t$-test). TST, total sleep time; SL, sleep latency; SE, sleep efficiency.

${ }^{*} P<0.05$.

the placebo arm than in the active treatment arm of a placebocontrolled trial because of the lack of efficacy of placebo, causing patients to leave the study prematurely [59].

Regarding basic research into the treatment of insomnia, current pharmacological approaches focus primarily on GABA, the major inhibitory neurotransmitter in the central nervous system [60]. SZRT was shown to increase non-REM sleep in an experimental model in rats, and the mechanism was thought to involve the stimulation of
$\mathrm{GABA}_{\mathrm{A}}$ and serotonin receptors $[44,45]$. Suanzaoren $(Z$. spinosa) was the chief ingredient of SZRT. It has been reported to have a sedative effect at higher doses and an anxiolytic effect at lower doses in animal studies [61]. In addition, there are some components of Suanzaoren that may have sedative-hypnotic effects. Jujuboside $A$ is a main component of jujubogenin extracted from the seed of Suanzaoren, which produces its sedative-hypnotic effects probably through its anticalmodulin action and inhibiting 
TABLE 3: Mean and change in BAI, BDI, and heroin craving score.

\begin{tabular}{|c|c|c|c|c|c|}
\hline & \multicolumn{2}{|c|}{ SZRT group $(n=45)$} & \multicolumn{2}{|c|}{ Placebo group $(n=45)$} & \multirow{2}{*}{$P$ value $^{\mathrm{a}}$} \\
\hline & Mean (SD) & Change & Mean (SD) & Change & \\
\hline \multicolumn{6}{|l|}{ BAI } \\
\hline Baseline & $15.0(11.3)$ & & $12.4(13.9)$ & & \\
\hline 4-week posttreatment & $12.8(13.2)$ & -2.2 & $8.8(13.0)$ & -3.6 & 0.502 \\
\hline \multicolumn{6}{|l|}{ BDI } \\
\hline Baseline & $18.3(14.3)$ & & $14.4(14.4)$ & & \\
\hline 4-week posttreatment & $13.0(13.9)$ & -5.3 & $11.5(13.3)$ & -2.9 & 0.280 \\
\hline \multicolumn{6}{|l|}{ Heroin craving score } \\
\hline Baseline & $25.5(28.1)$ & & $33.7(34.6)$ & & \\
\hline 4-week posttreatment & $33.5(31.9)$ & 8.3 & $34.2(35.6)$ & 0.68 & 0.351 \\
\hline
\end{tabular}

the glutamate-mediated excitatory signaling pathway in the hippocampus [62]. Saponins, isolated from Suanzaoren, can prolong the sleep time induced by barbiturates [63]. Sanjoinine $\mathrm{A}$ is one of major alkaloid compounds of Suanzaoren, which have anxiolytic-like effects, increase sleep time, and decrease sleep latency induced by pentobarbital, and these effects may be mediated by GABAergic transmission [64, 65]. Other ingredients of SZRT, including Fuling ( $P$. cocos), have been reported to enhance non-REM sleep by enhancing the secretion of cytokines, such as interleukin-1 $\beta$ and tumor necrotic factor- $\alpha$, in human peripheral blood monocytes $[66,67]$. Gancao (liquorice extract) has been shown to have an antidepressant-like effect that seems to be mediated by increase of brain norepinephrine and dopamine, but not by increase of serotonin in a murine model [68]. In a clinical trial, SZRT appeared to be a relatively safe and effective shortterm therapeutic option for improving daytime function of climacteric women with poor sleep quality [49].

In 2012, two studies $[69,70]$ reported the treatment for methadone-maintained patients with sleep disturbance. The baseline of the mean PSQI total score in the two studies $[69,70]$ was similar to our study. Li et al. [69] used acupuncture therapy for their patients. Acupuncture was applied to Baihui (GV20), Shenmen (bilateral, TF4), Shenting (GV24), Sanyinjiao (bilateral, SP6), and Sishencong (EX-HN1). They found that acupuncture treatment produced a statistically significant improvement in the mean total PSQI scores after four weeks of treatment [69]. Stein et al. reported trazodone did not improve subjective or objective sleep in methadonemaintained patients with sleep disturbance [70]. In addition, a previous study evaluated the effectiveness of melatonin in attenuating sleep difficulties during BZD withdrawal among patients in methadone maintenance treatment [71]. They found that melatonin improved sleep quality in patients who did not stop BZD but did not enhance BZD discontinuation [71].

Psychiatric comorbidity between anxiety, depression, and substance abuse is common. The prevalence of psychiatric disorder is up to 10 times higher in the methadonemaintained patients than in the general population [72]. At least one anxiety disorder in 55\% and affective disorder in $58 \%$ were found in the population receiving methadone maintenance treatment [73]. In our study, the average BAI scores in the baseline and posttreatment were 15.0 and 12.8 which both indicated mild anxiety. The average BDIII scores in the baseline and posttreatment were 18.3 and 13.0 which indicated mild depression in the baseline and minimal depression after treatment. In Asia, Suanzaoren has been prescribed for the treatment of depression, insomnia, and anxiety. The possible mechanism of action involves the serotonergic, noradrenergic, and monoamine oxidase enzyme system $[61,74]$. However, both BAI and BDI-II scores did not differ significantly between the groups after treatment in our study. Long-term treatment and bigger sample size for assessing the antidepressant and anxiolytic effect of SZRT should be further investigated.

A major limitation of our study is lack of an objective measurement, such as polysomnography. A previous study comparing subjective ratings, sleep diaries, and home polysomnography in methadone-maintained patients showed that subjective and objective sleep durations were similar in their sample, and average diary sleep time, subjective ratings of feeling rested, and polysomnography sleep efficiency were correlated significantly with PSQI score [75]. Therefore, the subjective measures used in this study may be indicative of objective sleep measures in methadonemaintained patients. The study is also limited by participant self-reporting, which may exaggerate the severity of sleep problems. Another limitation is the sleep efficiency calculation; typically sleep efficiency comparisons are based on a standard time in bed values, generally 480 minutes in sleep laboratory studies. A longer or shorter total time in bed can result in different sleep efficiency. In our study, we do not receive a standardized time in bed. At baseline of the total time in bed, the SZRT group was less than placebo group more than 30 minutes. In the 4 -week posttreatment assessment, the SZRT group still had shorter total time in bed than the placebo group. But there was no significant difference between two groups in the total time in bed. However, a shorter total time in bed can result in a relatively high sleep efficiency. This difference complicates the sleep efficiency comparison and the claim of a significant difference. In addition, participants were all from Taiwan; there needs to be caution in generalizing these findings to 
patients in other nations. Finally, although patients provided self-reports regarding current illicit drug use, the validity of these reports was not confirmed with urine toxicological data. Methadone-maintained patients have many ongoing risks for chronic insomnia and have high rates of use of other illicit drugs (e.g., amphetamine), cigarettes, or alcohol that can interfere with sleep.

Despite the limitations, this study retains a number of noteworthy strengths. The use of TCM for insomnia can be traced back more than 2000 years in Chinese medical texts. Notwithstanding the popularity of SZRT in the markets of China, Japan, South Korea, and Taiwan, this is the first evaluation of SZRT in opiate-dependent patients by a doubleblind, placebo-controlled, randomized clinical trial. The 4week courses of the SZRT treatment show it to be a welltolerated and valuable alternative therapeutic option for improving sleep quality in methadone-maintained patients.

\section{Conclusion}

Our data demonstrate that SZRT is effective in improving sleep quality and sleep efficiency among methadone-maintained patients with sleep complaints and appears safe in combination with methadone.

\author{
Abbreviations \\ MMT: Methadone maintenance therapy \\ PSQI: Pittsburgh Sleep Quality Index \\ REM: Rapid eye movement \\ BZD: Benzodiazepines \\ TCM: Traditional Chinese medicine \\ SZRT: Suan Zao Ren Tang \\ GABA: Gamma-aminobutyric acid \\ BAI: Beck Anxiety Inventory \\ BDI-II: Beck Depression Inventory II \\ TST: Total sleep time \\ SOL: Sleep onset latency \\ SE: $\quad$ Sleep efficiency \\ VAS: Visual analog scale.
}

\section{Disclosure}

The funder had no role in study design, data collection and analysis, decision to publish, or preparation of the manuscript.

\section{Conflict of Interests}

The authors declare that they have no competing interests.

\section{Acknowledgments}

This work was supported by Grants DMR-100-052 from CMUH and NSC 102-2320-B-039-024- from the National Science Council, Taiwan.

\section{References}

[1] Y.-I. Hser, V. Hoffman, C. E. Grella, and M. D. Anglin, "A 33-year follow-up of narcotics addicts," Archives of General Psychiatry, vol. 58, no. 5, pp. 503-508, 2001.

[2] A. T. McLellan, D. C. Lewis, C. P. O’Brien, and H. D. Kleber, "Drug dependence, a chronic medical illness implications for treatment, insurance, and outcomes evaluation," Journal of the American Medical Association, vol. 284, no. 13, pp. 1689-1695, 2000.

[3] V.P. Dole and M. Nyswander, "A medical treatment for diacetylmorphine (heroin) addiction. A clinical trial with methadone hydrochloride," Journal of the American Medical Association, vol. 193, pp. 646-650, 1965.

[4] V. P. Dole and M. E. Nyswander, "Rehabilitation of heroin addicts after blockade with methadone," New York State Journal of Medicine, vol. 66, no. 15, pp. 2011-2017, 1966.

[5] V. P. Dole and M. Nyswander, "Study of methadone as an adjunct in rehabilitation of heroin addicts," The Illinois Medical Journal, vol. 130, no. 4, pp. 487-489, 1966.

[6] "Position statement on methadone maintenance treatment," The American Journal of Psychiatry, vol. 151, no. 5, pp. 792-794, 1994.

[7] J. Ward, W. Hall, and R. P. Mattick, "Role of maintenance treatment in opioid dependence," The Lancet, vol. 353, no. 9148, pp. 221-226, 1999.

[8] A. Oyefeso, P. Sedgwick, and H. Ghodse, "Subjective sleepwake parameters in treatment-seeking opiate addicts," Drug and Alcohol Dependence, vol. 48, no. 1, pp. 9-16, 1997.

[9] E. Peles, S. Schreiber, and M. Adelson, "Variables associated with perceived sleep disorders in methadone maintenance treatment (MMT) patients," Drug and Alcohol Dependence, vol. 82, no. 2, pp. 103-110, 2006.

[10] M. D. Stein, D. S. Herman, S. Bishop et al., "Sleep disturbances among methadone maintained patients," Journal of Substance Abuse Treatment, vol. 26, no. 3, pp. 175-180, 2004.

[11] C. K. Burke, J. M. Peirce, M. S. Kidorf et al., "Sleep problems reported by patients entering opioid agonist treatment," Journal of Substance Abuse Treatment, vol. 35, no. 3, pp. 328-333, 2008.

[12] K. M. Sharkey, M. E. Kurth, B. J. Anderson, R. P. Corso, R. P. Millman, and M. D. Stein, "Obstructive sleep apnea is more common than central sleep apnea in methadone maintenance patients with subjective sleep complaints," Drug and Alcohol Dependence, vol. 108, no. 1-2, pp. 77-83, 2010.

[13] Y. Liao, J. Tang, T. Liu, X. Chen, T. Luo, and W. Hao, "Sleeping problems among Chinese heroin-dependent individuals," The American Journal of Drug and Alcohol Abuse, vol. 37, no. 3, pp. 179-183, 2011.

[14] M. B. Stein, S.-L. Belik, F. Jacobi, and J. Sareen, "Impairment associated with sleep problems in the community: relationship to physical and mental health comorbidity," Psychosomatic Medicine, vol. 70, no. 8, pp. 913-919, 2008.

[15] T. J. Balkin, T. Rupp, D. Picchioni, and N. J. Wesensten, "Sleep loss and sleepiness: current issues," Chest, vol. 134, no. 3, pp. 653-660, 2008.

[16] N. Solowij, R. Stephens, R. A. Roffman, and T. Babor, "Does marijuana use cause long-term cognitive deficits?" The Journal of the American Medical Association, vol. 287, no. 20, pp. 26532654, 2002.

[17] K. M. Sharkey, M. E. Kurth, R. P. Corso, K. J. Brower, R. P. Millman, and M. D. Stein, "Home polysomnography in 
methadone maintenance patients with subjective sleep complaints," The American Journal of Drug and Alcohol Abuse, vol. 35, no. 3, pp. 178-182, 2009.

[18] D. Wang and H. Teichtahl, "Opioids, sleep architecture and sleep-disordered breathing," Sleep Medicine Reviews, vol. 11, no. 1, pp. 35-46, 2007.

[19] H. Teichtahl, A. Prodromidis, B. Miller, G. Cherry, and I. Kronborg, "Sleep-disordered breathing in stable methadone programme patients: a pilot study," Addiction, vol. 96, no. 3, pp. 395-403, 2001.

[20] D. Wang, H. Teichtahl, O. Drummer et al., "Central sleep apnea in stable methadone maintenance treatment patients," Chest, vol. 128, no. 3, pp. 1348-1356, 2005.

[21] L. R. Webster, Y. Choi, H. Desai, L. Webster, and B. J. B. Grant, "Sleep-disordered breathing and chronic opioid therapy," Pain Medicine, vol. 9, no. 4, pp. 425-432, 2008.

[22] R. Lydic and H. A. Baghdoyan, "Sleep, anesthesiology, and the neurobiology of arousal state control," Anesthesiology, vol. 103, no. 6, pp. 1268-1295, 2005.

[23] C. J. Watson, R. Lydic, and H. A. Baghdoyan, "Sleep and GABA levels in the oral part of rat pontine reticular formation are decreased by local and systemic administration of morphine," Neuroscience, vol. 144, no. 1, pp. 375-386, 2007.

[24] A. M. Nelson, A. S. Battersby, H. A. Baghdoyan, and R. Lydic, "Opioid-induced decreases in rat brain adenosine levels are reversed by inhibiting adenosine deaminase," Anesthesiology, vol. 111, no. 6, pp. 1327-1333, 2009.

[25] G. H. Trksak, J. E. Jensen, D. T. Plante et al., "Effects of sleep deprivation on sleep homeostasis and restoration during methadone-maintenance: a ${ }^{[31]} \mathrm{P}$ MRS brain imaging study," Drug and Alcohol Dependence, vol. 106, no. 2-3, pp. 79-91, 2010.

[26] National Institutes of Health State, "National Institutes of Health State of the Science Conference statement on manifestations and management of chronic insomnia in adults, June 13-15, 2005," Sleep, vol. 28, no. 9, pp. 1049-1057, 2005.

[27] H. Ashton, "The diagnosis and management of benzodiazepine dependence," Current Opinion in Psychiatry, vol. 18, no. 3, pp. 249-255, 2005.

[28] S. E. Lankenau, B. Sanders, J. J. Bloom et al., "Prevalence and patterns of prescription drug misuse among young ketamine injectors," Journal of Drug Issues, vol. 37, no. 3, pp. 717-736, 2007.

[29] K. W. Chen, C. C. Berger, D. P. Forde, C. D’Adamo, E. Weintraub, and D. Gandhi, "Benzodiazepine use and misuse among patients in a methadone program," BMC Psychiatry, vol. 11, article 90, 2011.

[30] A. Bleich, M. Gelkopf, T. Weizman, and M. Adelson, "Benzodiazepine abuse in a methadone maintenance treatment clinic in Israel: characteristics and a pharmacotherapeutic approach," Israel Journal of Psychiatry and Related Sciences, vol. 39, no. 2, pp. 104-112, 2002.

[31] M. Y. Iguchi, L. Handelsman, W. K. Bickel, and R. R. Griffiths, "Benzodiazepine and sedative use/abuse by methadone maintenance clients," Drug and Alcohol Dependence, vol. 32, no. 3, pp. 257-266, 1993.

[32] E. Lavie, M. Fatséas, C. Denis, and M. Auriacombe, "Benzodiazepine use among opiate-dependent subjects in buprenorphine maintenance treatment: correlates of use, abuse and dependence," Drug and Alcohol Dependence, vol. 99, no. 1-3, pp. 338-344, 2009.
[33] F. Kamal, S. Flavin, F. Campbell, J. Fagan, C. Behan, and R. Smyth, "Factors affecting the outcome of methadone maintenance treatment in opiate dependence," Irish Medical Journal, vol. 100, no. 3, pp. 393-397, 2007.

[34] E. Peles, S. Schreiber, and M. Adelson, "15-year survival and retention of patients in a general hospital-affiliated methadone maintenance treatment (MMT) center in Israel," Drug and Alcohol Dependence, vol. 107, no. 2-3, pp. 141-148, 2010.

[35] S. Kanba, K. Yamada, H. Mizushima, and M. Asai, "Use of herbal medicine for treating psychiatric disorders in Japan," Psychiatry and Clinical Neurosciences, vol. 52, pp. S331-S333, 1998.

[36] Y.-I. Shin, C.-Y. Yang, M.-C. Joo, S.-G. Lee, J.-H. Kim, and M. S. Lee, "Patterns of using complementary and alternative medicine by stroke patients at two university hospitals in Korea," Evidence-Based Complementary and Alternative Medicine, vol. 5, no. 2, pp. 231-235, 2008.

[37] G. J. Dobos, L. Tan, M. H. Cohen et al., "Are national quality standards for traditional Chinese herbal medicine sufficient? Current governmental regulations for traditional Chinese herbal medicine in certain Western countries and China as the Eastern origin country," Complementary Therapies in Medicine, vol. 13, no. 3, pp. 183-190, 2005.

[38] F.-P. Chen, T.-J. Chen, Y.-Y. Kung et al., "Use frequency of traditional Chinese medicine in Taiwan," BMC Health Services Research, vol. 7, article 26, 2007.

[39] H. C. Chen and M. T. Hsieh, "Clinical trial of suanzaorentang in the treatment of insomnia," Clinical Therapeutics, vol. 7, no. 3, pp. 334-337, 1985.

[40] R. Aizawa, T. Kanbayashi, Y. Saito et al., "Effects of Yokukan-san-ka-chimpi-hange on the sleep of normal healthy adult subjects," Psychiatry and Clinical Neurosciences, vol. 56, no. 3, pp. 303-304, 2002.

[41] Y. Yang, H. Li, S. Zhang et al., "TCM treatment for 63 cases of senile dyssomnia," Journal of Traditional Chinese Medicine, vol. 25, no. 1, pp. 45-49, 2005.

[42] F. P. Chen, M. S. Jong, Y. C. Chen et al., "Prescriptions of Chinese herbal medicines for Insomnia in Taiwan during 2002," Evidence-Based Complementary and Alternative Medicine, vol. 2011, Article ID 236341, 9 pages, 2011.

[43] K. F. Chung and C. K. Y. Lee, "Over-the-counter sleeping pills: a survey of use in Hong Kong and a review of their constituents," General Hospital Psychiatry, vol. 24, no. 6, pp. 430-435, 2002.

[44] P.-L. Yi, C.-H. Tsai, Y.-C. Chen, and F.-C. Chang, "Gammaaminobutyric acid (GABA) receptor mediates suanzaorentang, a traditional Chinese herb remedy, -induced sleep alteration," Journal of Biomedical Science, vol. 14, no. 2, pp. 285-297, 2007.

[45] P.-L. Yi, C.-P. Lin, C.-H. Tsai, J.-G. Lin, and F.-C. Chang, "The involvement of serotonin receptors in suanzaorentang-induced sleep alteration," Journal of Biomedical Science, vol. 14, no. 6, pp. 829-840, 2007.

[46] J. Sarris and G. J. Byrne, "A systematic review of insomnia and complementary medicine," Sleep Medicine Reviews, vol. 15, no. 2, pp. 99-106, 2011.

[47] J. Sarris, A. Panossian, I. Schweitzer, C. Stough, and A. Scholey, "Herbal medicine for depression, anxiety and insomnia: a review of psychopharmacology and clinical evidence," European Neuropsychopharmacology, vol. 21, no. 12, pp. 841-860, 2011.

[48] W.-F. Yeung, K.-F. Chung, M. M. Poon et al., "Chinese herbal medicine for insomnia: a systematic review of randomized 
controlled trials," Sleep Medicine Reviews, vol. 16, no. 6, pp. 497507, 2012.

[49] C.-H. Yeh, C. K. Arnold, Y.-H. Chen, and J.-N. Lai, "Suan Zao Ren Tang as an original treatment for sleep difficulty in climacteric women: a prospective clinical observation," Evidence-Based Complementary and Alternative Medicine, vol. 2011, Article ID 673813, 8 pages, 2011.

[50] D. J. Buysse, C. F. Reynolds III, T. H. Monk, S. R. Berman, and D. J. Kupfer, "The Pittsburgh sleep quality index: a new instrument for psychiatric practice and research," Psychiatry Research, vol. 28, no. 2, pp. 193-213, 1989.

[51] P.-S. Tsai, S.-Y. Wang, M.-Y. Wang et al., "Psychometric evaluation of the Chinese version of the Pittsburgh Sleep Quality Index (CPSQI) in primary insomnia and control subjects," Quality of Life Research, vol. 14, no. 8, pp. 1943-1952, 2005.

[52] K. A. Thomas and R. L. Burr, "Accurate assessment of mother \& infant sleep: how many diary days are required?" MCN The American Journal of Maternal/Child Nursing, vol. 34, no. 4, pp. 256-260, 2009.

[53] A. T. Beck, N. Epstein, G. Brown, and R. A. Steer, "An inventory for measuring clinical anxiety: psychometric properties," Journal of Consulting and Clinical Psychology, vol. 56, no. 6, pp. 893-897, 1988.

[54] A. T. Beck and R. A. Steer, "Internal consistencies of the original and revised Beck Depression Inventory," Journal of Clinical Psychology, vol. 40, no. 6, pp. 1365-1367, 1984.

[55] M. E. Wewers and N. K. Lowe, "A critical review of visual analogue scales in the measurement of clinical phenomena," Research in nursing \& health, vol. 13, no. 4, pp. 227-236, 1990.

[56] K. J. Brower and B. E. Perron, "Sleep disturbance as a universal risk factor for relapse in addictions to psychoactive substances," Medical Hypotheses, vol. 74, no. 5, pp. 928-933, 2010.

[57] R. M. Benca and M. J. Peterson, "Insomnia and depression," Sleep Medicine, vol. 9, supplement 1, pp. S3-S9, 2008.

[58] S. Mineka, D. Watson, and L. A. Clark, "Comorbidity of anxiety and unipolar mood disorders," Annual Review of Psychology, vol. 49, pp. 377-412, 1998.

[59] G. Kemmler, M. Hummer, C. Widschwendter, and W. W. Fleischhacker, "Dropout rates in placebo-controlled and activecontrol clinical trials of antipsychotic drugs: a meta-analysis," Archives of General Psychiatry, vol. 62, no. 12, pp. 1305-1312, 2005.

[60] B. Ebert, K. A. Wafford, and S. Deacon, "Treating insomnia: current and investigational pharmacological approaches," Pharmacology and Therapeutics, vol. 112, no. 3, pp. 612-629, 2006.

[61] W.-H. Peng, M.-T. Hsieh, Y.-S. Lee, Y.-C. Lin, and J. Liao, "Anxiolytic effect of seed of Ziziphus jujuba in mouse models of anxiety," Journal of Ethnopharmacology, vol. 72, no. 3, pp. 435441, 2000.

[62] M. Zhang, G. Ning, C. Shou, Y. Lu, D. Hong, and X. Zheng, "Inhibitory effect of jujuboside A on glutamate-mediated excitatory signal pathway in hippocampus," Planta Medica, vol. 69, no. 8, pp. 692-695, 2003.

[63] J.-G. Jiang, X.-J. Huang, and J. Chen, "Separation and purification of saponins from Semen Ziziphus jujuba and their sedative and hypnotic effects," Journal of Pharmacy and Pharmacology, vol. 59, no. 8, pp. 1175-1180, 2007.

[64] Y. Ma, H. Han, J. S. Eun, H.-C. Kim, J.-T. Hong, and K.-W. Oh, "Sanjoinine A isolated from Zizyphi Spinosi Semen augments pentobarbital-induced sleeping behaviors through the modification of GABA-ergic systems," Biological and Pharmaceutical Bulletin, vol. 30, no. 9, pp. 1748-1753, 2007.
[65] H. Han, Y. Ma, J. S. Eun et al., "Anxiolytic-like effects of sanjoinine A isolated from Zizyphi Spinosi Semen: possible involvement of GABAergic transmission," Pharmacology Biochemistry and Behavior, vol. 92, no. 2, pp. 206-213, 2009.

[66] S.-J. Yu and J. Tseng, "Fu-Ling, a Chinese herbal drug, modulates cytokine secretion by human peripheral blood monocytes," International Journal of Immunopharmacology, vol. 18, no. 1, pp. 37-44, 1996.

[67] J. M. Krueger, J. Fang, P. Taishi, Z. Chen, T. Kushikata, and J. Gardi, "Sleep. A physiologic role for IL-1 beta and TNF-alpha," Annals of the New York Academy of Sciences, vol. 856, pp. 148159,1998

[68] D. Dhingra and A. Sharma, "Antidepressant-like activity of Glycyrrhiza glabra L. in mouse models of immobility tests," Progress in Neuro-Psychopharmacology and Biological Psychiatry, vol. 30, no. 3, pp. 449-454, 2006.

[69] Y. Li, X.-B. Liu, and Y. Zhang, "Acupuncture therapy for the improvement of sleep quality of outpatients receiving methadone maintenance treatment: a randomized controlled trial," Zhongguo Zhong Xi Yi Jie He Za Zhi, vol. 32, no. 8, pp. 1056-1059, 2012.

[70] M. D. Stein, M. E. Kurth, K. M. Sharkey, B. J. Anderson, R. P. Corso, and R. P. Millman, "Trazodone for sleep disturbance during methadone maintenance: a double-blind, placebocontrolled trial," Drug and Alcohol Dependence, vol. 120, no. 1-3, pp. 65-73, 2012.

[71] E. Peles, T. Hetzroni, R. Bar-Hamburger, M. Adelson, and S. Schreiber, "Melatonin for perceived sleep disturbances associated with benzodiazepine withdrawal among patients in methadone maintenance treatment: a double-blind randomized clinical trial," Addiction, vol. 102, no. 12, pp. 1947-1953, 2007.

[72] T. Callaly, T. Trauer, L. Munro, and G. Whelan, "Prevalence of psychiatric disorder in a methadone maintenance population," Australian and New Zealand Journal of Psychiatry, vol. 35, no. 5, pp. 601-605, 2001.

[73] J. B. Milby, M. K. Sims, S. Khuder et al., "Psychiatric comorbidity: prevalence in methadone maintenance treatment," The American Journal of Drug and Alcohol Abuse, vol. 22, no. 1, pp. 95-107, 1996.

[74] J. Liu, W. Qiao, Y. Yang, L. Ren, Y. Sun, and S. Wang, "Antidepressant-like effect of the ethanolic extract from Suanzaorenhehuan Formula in mice models of depression," Journal of Ethnopharmacology, vol. 141, no. 1, pp. 257-264, 2012.

[75] K. M. Sharkey, M. E. Kurth, B. J. Anderson, R. P. Corso, R. P. Millman, and M. D. Stein, "Assessing sleep in opioid dependence: a comparison of subjective ratings, sleep diaries, and home polysomnography in methadone maintenance patients," Drug and Alcohol Dependence, vol. 113, no. 2-3, pp. 245-248, 2011. 


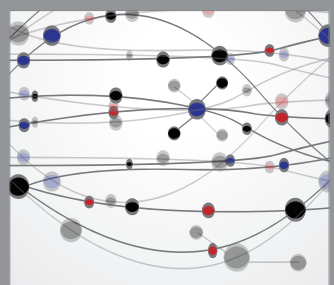

The Scientific World Journal
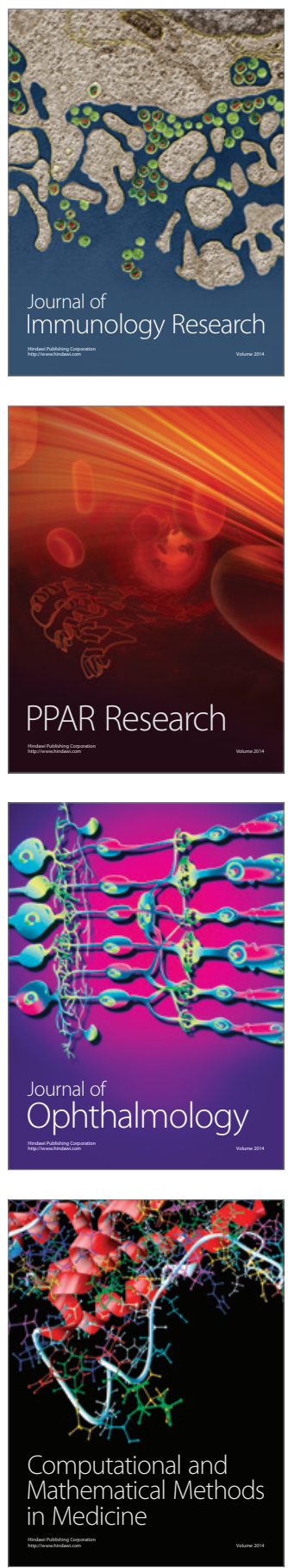

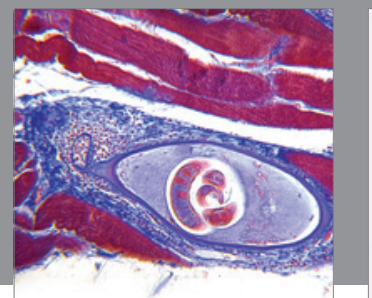

Gastroenterology

Research and Practice
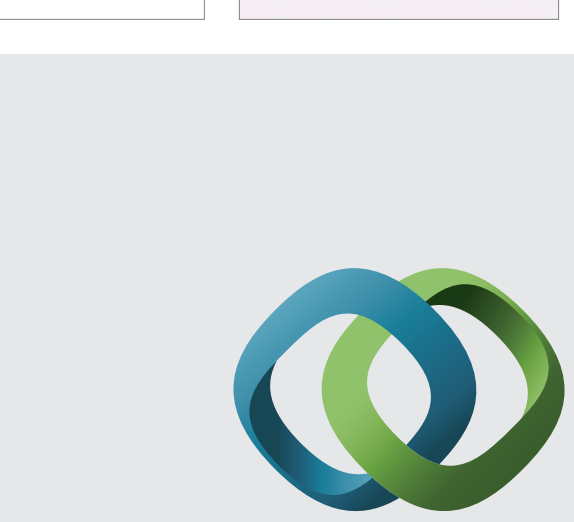

\section{Hindawi}

Submit your manuscripts at

http://www.hindawi.com
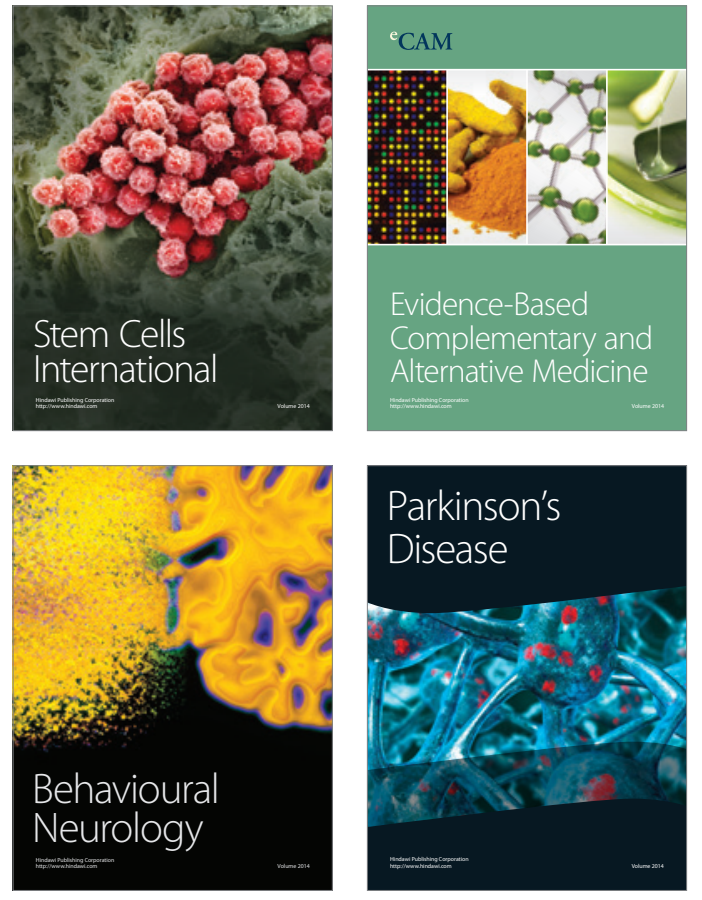
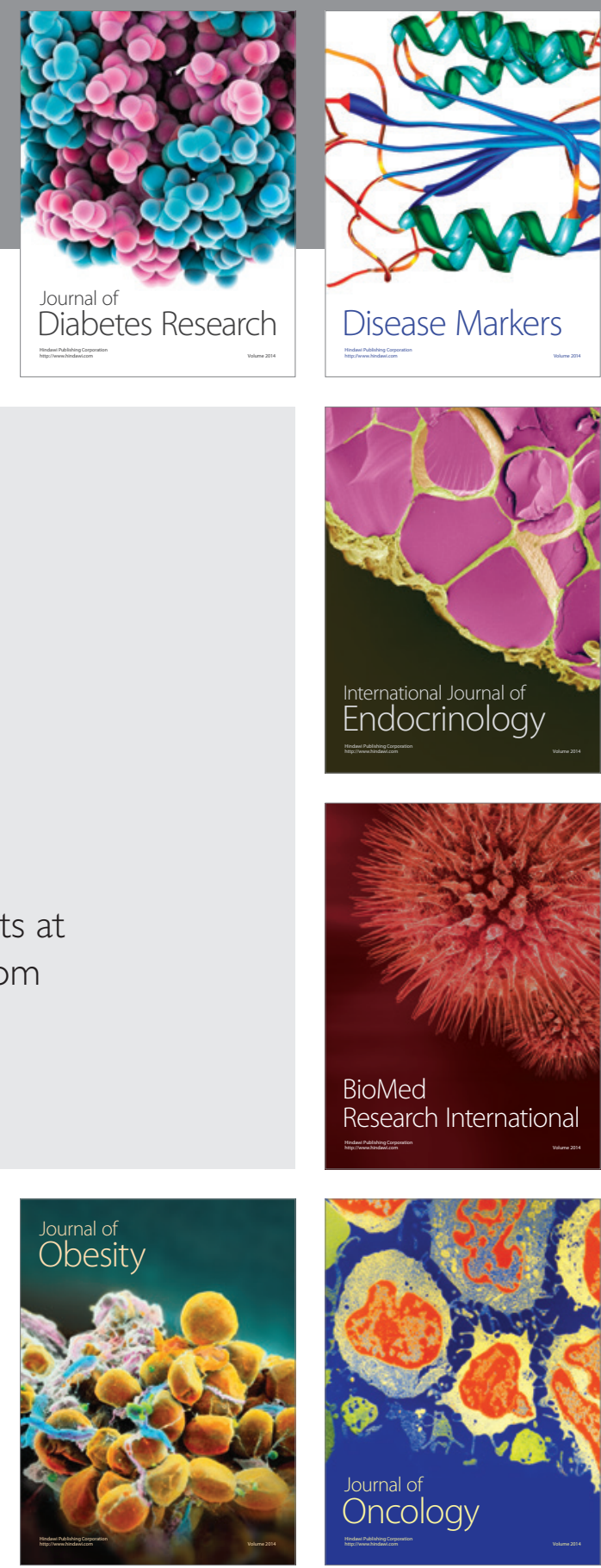

Disease Markers
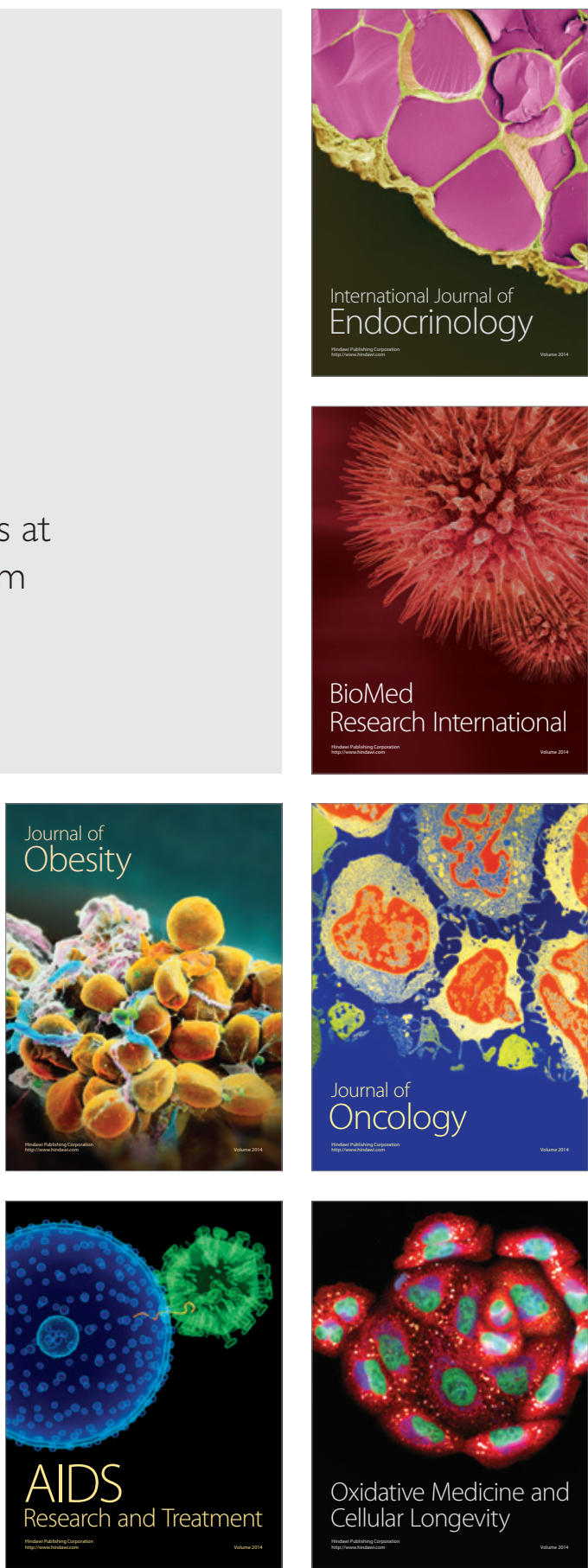